\title{
A New Remote User Authentication Scheme based on Graphical Password using Smart Card
}

\author{
Khanjan Ch. Baruah, Subhasish Banerjee, Manash P. Dutta and Chandan T. \\ Bhunia \\ Department of Computer Science and Engineering \\ National Institute of Technology, Arunachal Pradesh, India - 791112 \\ khanjan099@yahoo.com,subhasish.cse@nitap.in, \\ manash.cse@nitap.in,ctbhunia@vsnl.com
}

\begin{abstract}
Remote user authentication schemes provide a system to verify the legitimacy of remote users' login request over insecure communication channel. Since last few years many authentication schemes have been proposed including several new features and ideas. But, due to the advancement of computational process they are suffering from various possible attacks. This is the reason because of which attaining the security becomes a prime issue and major challenge among the researchers. In this paper, we have proposed a new user friendly authentication scheme that can provide the higher security at lower computational cost. This scheme provides extra level of security by adopting the userdefined image for password generation. Finally, the security analysis and performance evaluation are made to proof the efficiency of our scheme.
\end{abstract}

Keywords: Mutual Authentication, Multi-server, Dynamic ID

\section{Introduction}

Network technology has been widely developed in the recent years. This development brings many new issues of concern. Remote user authentication is one of the fundamental criteria in the network security. Researchers, in the past, devised many authentication schemes. The traditional schemes were based on passwords [1-3], making it vulnerable to simple dictionary attacks. Thereafter, to increase the security, smart card based authentication schemes were introduced [4-6]. But, due to the advancement of computer technology, these schemes are still not capable to withstand various possible attacks [7]. To overcome these weaknesses, later biometrics was also been incorporated as third parameter and make the authentication schemes [8-18] based on three factors namely smartcard, password and biometric. In 2010, Li and Hwang [10] proposed a biometricsbased remote user authentication scheme using smart cards. In their scheme, they eliminated the use of time synchronization by using nonce. Li et al. [11], in 2011, analyzed Li and Hwang's [10] scheme and drew various weaknesses. Subsequently, they proposed their improved authentication scheme to overcome drawbacks of $\mathrm{Li}$ and Hwang's authentication scheme. Later, in 2011, Das et al. [12] showed that Li et al.'s [11] scheme failed to locally update user's new password and also failed to provide strong mutual authentication. Thus, they proposed an efficient biometric-based remote user authentication scheme based on smart cards. In 2012, Sekhar et al. [13] and An et al. [14] analyzed Das et al.'s [12] scheme and showed that it was vulnerable to insider attack, impersonation attack, server masquerade attack, Man-in-the-Middle attack, and replay attack. An et al. [14] proposed a scheme which was resilient to all the vulnerabilities found in Das et al.'s scheme. In 2013, Khan et al. [15] showed that An et al.'s [14] scheme was not secure enough as they claimed and can suffer from impersonation attack, server masquerade attack, Man in the Middle attack. To overcome from all the mentioned 
attacks, they proposed their improved scheme by negotiating the computational cost by adding only two hash operations. In 2014, Chuang and Chen [16] proposed a multi- server authenticated key agreement scheme using smart cards and biometrics. However, both Mishra et al. [17] and Maitra and Giri [18] showed their scheme is insecure against stolen smart card attack, impersonation attack, forgery attack and session key discloser attack. Later, Mishra et al. [17] proposed an improved biometric based remote user authentication scheme. But, normally human brain is better in recalling images based things rather than remembering complicated large password. So, in this paper, we have proposed a user friendly remote user authentication scheme using smart card. In our proposed scheme, the users need not to remember any complicated alphanumeric password for security concern as because password can be generated automatically by selecting some regions from an image provided by the user itself during registration. The latter half of the paper consists of the security and performance analysis of the proposed scheme.

Table 1. Notation Used in the Paper

\begin{tabular}{|l|l|}
\hline Notations & Meaning \\
\hline $\mathrm{ID}_{\mathrm{i}}$ & Identity of the $\mathrm{i}^{\text {th }}$ user \\
\hline $\mathrm{RC}$ & Registration center \\
\hline $\mathrm{SID}_{\mathrm{j}}$ & Identity of the $\mathrm{j}^{\text {th }}$ server \\
\hline $\mathrm{PW}_{\mathrm{i}}$ & Password of the $\mathrm{i}^{\text {th }}$ user \\
\hline $\mathrm{IMG}$ & Image provided by the user during registration \\
\hline $\mathrm{PSK}$ & Pre-shared key of the servers \\
\hline $\mathrm{X}_{\mathrm{s}}$ & $\begin{array}{l}\text { Master secret key maintained by the registration } \\
\text { center }\end{array}$ \\
\hline $\mathrm{h}()$. & A one-way hash function \\
\hline $\mathrm{N}_{\mathrm{i}}$ & Random nonce of the $\mathrm{i}^{\text {th }}$ user \\
\hline $\mathrm{N}_{\mathrm{j}}$ & Random nonce of the $\mathrm{j}^{\text {th }}$ server \\
\hline$\oplus$ & Exclusive-OR operation \\
\hline$\|$ & Message concatenation operation \\
\hline
\end{tabular}

\section{Password Generation}

Password is the important factor in authentication scheme. The traditional authentication schemes require the user to input text-based password. However, these passwords are weak or are easily forgotten. It was found that human can remember pictures easier than text.

In our proposed scheme, the user's password is generated by using an image provided by the user. During registration, the user provides an image IMG. The image is reduced to a size of $\mathrm{m} \times \mathrm{m}$ pixels (say $640 \times 640$ pixels) and converted to greyscale image. The image is then divided into $\mathrm{n}$ blocks of $1 \times 1$ size (say $8 \times 8$ pixels). The user, now selects $\mathrm{k}$ number of blocks from the greyscale image. These blocks have individual pixels with each pixel having some value. Now, we add every pixel value to obtain the block value. The block values of the selected $\mathrm{k}$ blocks are further added to give us the password of the user. The user can change the image or the password as and when required. Figure 1 graphically represents this process.

$$
P W_{i}=\sum_{j=1}^{k} \text { Block }_{j}
$$




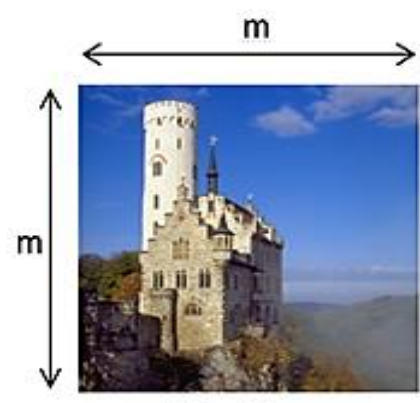

Original Image

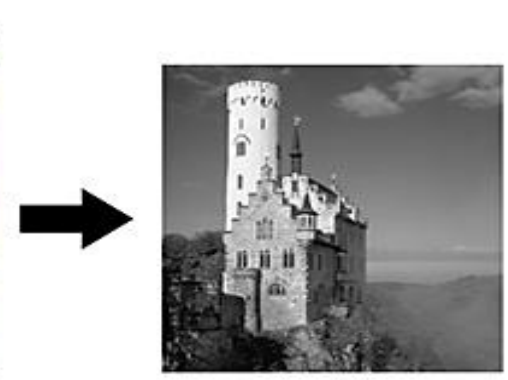

Greyscale Image

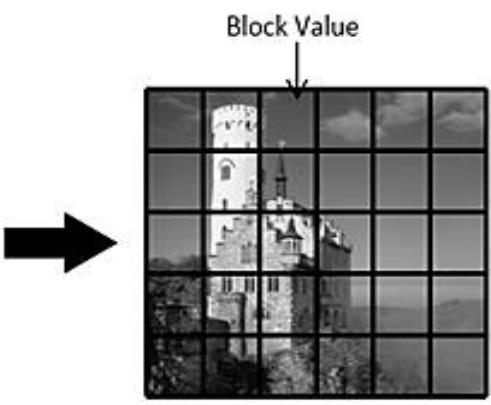

Figure 1. Password Generation from an Image

\section{Proposed Scheme}

In this section, we have proposed a remote user authentication scheme using smart card. Typically, three participants are involved in the exchange of messages among one another. These participants are: the user (with identity IDi), the server (with identity $\operatorname{SID}_{j}$ ) and the registration center (with identity RC). The proposed scheme has been designed to work with the following phases namely registration, login, authentication and password change phase. The notations which have been used throughout this paper are listed in Table 1.

\subsection{Registration Phase}

The scheme starts with the registration of the users and the service provider servers with the registration center. In this phase, the user performs the following steps to complete his registration:

1) The user, with identity $\mathrm{ID}_{\mathrm{i}}$, selects an image IMG. The password $\mathrm{PW}_{\mathrm{i}}$ is generated from this image through the process mentioned in Section II.

2) The user generates a random number $R_{i}$ and computes $U_{1}=h\left(R_{i}|| P W_{i}\right)$ and $U_{2}=$ $\mathrm{h}\left(\mathrm{PW}_{\mathrm{i}} \oplus \mathrm{ID}_{\mathrm{i}}\right)$. The user sends their $\mathrm{ID}_{\mathrm{i}}, \mathrm{U}_{1}$ and $\mathrm{U}_{2}$ to the registration center via a secure channel.

3) After receiving the registration request message, registration center, $\mathrm{RC}$, computes

$\mathrm{A}_{\mathrm{i}}=\mathrm{h}\left(\mathrm{ID}_{\mathrm{i}} \| \mathrm{X}_{\mathrm{s}}\right)$

$\mathrm{B}_{\mathrm{i}}=\mathrm{A}_{\mathrm{i}} \oplus \mathrm{U}_{2}$

$\mathrm{C}_{\mathrm{i}}=\mathrm{PSK} \oplus \mathrm{h}\left(\mathrm{U}_{1} \| \mathrm{ID}_{\mathrm{i}}\right)$

and generates the smart card containing information $\mathrm{B}_{\mathrm{i}}$ and $\mathrm{C}_{\mathrm{i}}$. The smart card is then sent to the user.

4) The user, upon receiving the smart card, updates it with $D_{i}=h\left(I_{i} \| P W_{i}\right), I M G$ and $R_{i}$. Finally, the smart card will be updated by $\left\{B_{i}, C_{i}, D_{i}, I M G, R_{i}\right\}$.

Similarly, the server, who wants to provide his services, need to register itself to the registration center, $\mathrm{RC}$, to become an authorized server. It sends a join request to $\mathrm{RC}$ along with its identity $\mathrm{SID}_{\mathrm{j}}$. The $\mathrm{RC}$ replies with $\mathrm{h}\left(\mathrm{SID}_{\mathrm{j}}|| \mathrm{PSK}\right)$ and $\mathrm{X}_{\mathrm{s}}$, which the server uses to authenticate users.

\subsection{Login Phase}

This phase will be invoked whenever a registered user wants to access the registered service provider server. The complete description of this phase is defined below: 
1) The user $\mathrm{ID}_{\mathrm{i}}$ inserts his/her smart card $\mathrm{SC}_{\mathrm{i}}$ into the card reader and provides his/her identity $\mathrm{ID}_{\mathrm{i}}$. The system displays the image IMG stored in the smart card and prompts the user to select the blocks.

2) The user now selects specific blocks from IMG. Then, the terminal computes the password using the corresponding pixel value of the blocks as defined in the Section II.

3) The smart card computes the value $h\left(I_{i}|| P W_{i}\right)$ and verifies it with $D_{i}$. If the verification fails, the process terminates. Otherwise, the smart card computes

$\mathrm{U}_{1}=\mathrm{h}\left(\mathrm{R}_{\mathrm{i}} \| \mathrm{PW}_{\mathrm{i}}\right)$

$\mathrm{U}_{2}=\mathrm{h}\left(\mathrm{PW}_{\mathrm{i}} \oplus \mathrm{ID}_{\mathrm{i}}\right)$

The smart card extracts PSK and $\mathrm{A}_{\mathrm{i}}$ from $\mathrm{C}_{\mathrm{i}}$ and $\mathrm{B}_{\mathrm{i}}$ respectively by computing

$\mathrm{PSK}=\mathrm{C}_{\mathrm{i}} \oplus \mathrm{h}\left(\mathrm{U}_{1} \| \mathrm{ID}_{\mathrm{i}}\right)$

$\mathrm{A}_{\mathrm{i}}=\mathrm{B}_{\mathrm{i}} \oplus \mathrm{U}_{2}$

4) Using the PSK, it computes $\mathrm{h}\left(\mathrm{SID}_{\mathrm{j}} \| \mathrm{PSK}\right)$, where $\mathrm{SID}_{\mathrm{j}}$ is the server's identity to which it is trying to communicate. A message, using $\mathrm{h}\left(\mathrm{SID}_{\mathrm{j}} \| \mathrm{PSK}\right)$ and $\mathrm{ID}_{\mathrm{i}}$, is generated as : $\mathrm{M}_{1}=\mathrm{ID}_{\mathrm{i}} \oplus \mathrm{h}\left(\mathrm{SID}_{\mathrm{j}} \| \mathrm{PSK}\right)$.

5) A random number $N_{i}$ is generated by the smart card and it is sent to the server as: $M_{2}$ $=N_{i} \oplus A_{i}$. A message for verification is also generated as $M_{v}=h\left(S_{I D}\left\|N_{i}\right\| A_{i}\right)$.

6) The smart card transmits the login request messages $\left\{M_{1}, M_{2}, M_{v}\right\}$ to the server $\operatorname{SID}_{j}$ via an insecure channel.

\subsection{Authentication Phase}

The server $\operatorname{SID}_{j}$, upon receiving the authentication messages, $\left\{\mathrm{M}_{1}, \mathrm{M}_{2}, \mathrm{M}_{\mathrm{v}}\right\}$, performs the following operations to validate the user and mutually generate the session key.

1). The server extracts the identity $\mathrm{ID}_{\mathrm{i}}$ of the user from message $\mathrm{M}_{1}$ : $\mathrm{ID}_{\mathrm{i}}=\mathrm{M}_{1} \oplus \mathrm{h}\left(\mathrm{SID}_{\mathrm{j}} \| \mathrm{PSK}\right)$

2). The server now uses the user's identity, $I_{i}$ and its shared secret $X_{s}$ to compute $A_{i}$. This is further used to extract the user random number $\mathrm{N}_{\mathrm{i}}$ from the received message $\mathrm{M}_{2}$.

$\mathrm{A}_{\mathrm{i}}=\mathrm{h}\left(\mathrm{ID}_{\mathrm{i}} \| \mathrm{X}_{\mathrm{s}}\right)$

$\mathrm{N}_{\mathrm{i}}=\mathrm{M}_{2} \oplus \mathrm{A}_{\mathrm{i}}$

3). Then the server verifies whether $h\left(\operatorname{SID}_{j}\left\|N_{i}\right\| A_{i}\right)$ is matched with received message $M_{v}$ or not.

4). If verification fails, the server simply rejects the login request otherwise it computes the session key for further communication after generating the nonce $\mathrm{N}_{\mathrm{j}}$. $\mathrm{SK}_{\mathrm{ji}}=\mathrm{h}\left(\mathrm{A}_{\mathrm{i}}\left\|\mathrm{SID}_{\mathrm{j}}|| \mathrm{N}_{\mathrm{i}}\right\| \mathrm{N}_{\mathrm{j}}\right)$

5). The server information must be communicated to the actual user for the establishment of the session key agreement on the user side. So, the server generates the message $M_{3}$. The server verifies its authenticity to the user through the message $\mathbf{M}_{4}$.

$\mathrm{M}_{3}=\mathrm{N}_{\mathrm{j}} \oplus \mathrm{h}\left(\mathrm{ID}_{\mathrm{i}} \oplus \mathrm{N}_{\mathrm{i}}\right)$

$\mathrm{M}_{4}=\mathrm{h}\left(\mathrm{SK}_{\mathrm{ji}}\left\|\mathrm{N}_{\mathrm{j}}\right\| \mathrm{N}_{\mathrm{i}}\right)$

6). These messages $\mathrm{M}_{3}$ and $\mathrm{M}_{4}$ are forwarded to the user through an insecure channel.

7). After receiving the message $\left\{\mathrm{M}_{3}, \mathrm{M}_{4}\right\}$, the user extracts the server's random number $\mathrm{N}_{\mathrm{j}}$ from $\mathrm{M}_{3}$. $\mathrm{N}_{\mathrm{j}}=\mathrm{M}_{3} \oplus \mathrm{h}\left(\mathrm{ID}_{\mathrm{i}} \oplus \mathrm{N}_{\mathrm{i}}\right)$

8). The user computes the session key $\mathrm{SK}_{\mathrm{ij}}=\mathrm{h}\left(\mathrm{A}_{\mathrm{i}}|| \mathrm{SID}_{\mathrm{j}}|| \mathrm{N}_{\mathrm{i}}|| \mathrm{N}_{\mathrm{j}}\right)$.

9). The user verifies the server's authenticity by computing $h\left(\mathrm{SK}_{\mathrm{ji}}|| \mathrm{N}_{\mathrm{j}}|| \mathrm{N}_{\mathrm{i}}\right)$ and comparing it with $\mathrm{M}_{4}$. If successful, the user can conclude that the server is authentic and both of them have the same session key. 


\subsection{Password Change Phase}

The password change phase is a significant part of any authentication scheme. The password change mechanism can be performed locally without contacting the registration center.

The user inserts his/her smart card and inputs identity $\mathrm{ID}_{\mathrm{i}}$. The system prompts the user to select the $k$ blocks from the image, IMG, stored in the smart card. The card verifies the information entered using $\mathrm{D}_{\mathrm{i}}$. The user has two options either to input a new image or to select new blocks. The user selects the blocks and the password is generated as mentioned in Section II. The new password $\mathrm{PW}_{\mathrm{i}}^{\text {new }}$ is generated and the card computes:

$$
\begin{aligned}
& \mathrm{U}_{1}=\mathrm{h}\left(\mathrm{R}_{\mathrm{i}} \| \mathrm{PW}_{\mathrm{i}}\right) \\
& \mathrm{U}_{2}=\mathrm{h}\left(\mathrm{PW}_{\mathrm{i}} \oplus \mathrm{ID}_{\mathrm{i}}\right) \\
& \mathrm{U}_{1}{ }^{\text {new }}=\mathrm{h}\left(\mathrm{R}_{\mathrm{i}} \| \mathrm{PW}_{\mathrm{i}}{ }^{\text {new }}\right) \\
& \mathrm{U}_{2}{ }^{\text {new }}=\mathrm{h}\left(\mathrm{PW}_{\mathrm{i}}^{\text {new }} \oplus \mathrm{ID}_{\mathrm{i}}\right) \\
& \mathrm{B}_{\mathrm{i}}{ }^{\text {new }}=\mathrm{B}_{\mathrm{i}} \oplus \mathrm{U}_{2} \oplus \mathrm{U}_{2}{ }^{\text {new }} \\
& \mathrm{C}_{\mathrm{i}}{ }^{\text {new }}=\mathrm{C}_{\mathrm{i}} \oplus \mathrm{h}\left(\mathrm{U}_{1} \| \mathrm{ID}_{\mathrm{i}}\right) \oplus \mathrm{h}\left(\mathrm{U}_{1}{ }^{\text {new }} \| \mathrm{ID}_{\mathrm{i}}\right) \\
& \mathrm{D}_{\mathrm{i}}{ }^{\text {new }}=\mathrm{h}\left(\mathrm{ID}_{\mathrm{i}} \| \mathrm{PW}_{\mathrm{i}}{ }^{\text {new }}\right)
\end{aligned}
$$

The smart card replaces the old $B_{i}, C_{i}, D_{i}$ with the newly computed values, $B_{i}{ }^{\text {new }}, C_{i}^{\text {new }}$, $\mathrm{D}_{\mathrm{i}}^{\text {new }}$. Thus the updated smart card $\mathrm{SC}_{\mathrm{i}}=\left\{\mathrm{B}_{\mathrm{i}}^{\text {new }}, \mathrm{C}_{\mathrm{i}}^{\text {new }}, \mathrm{D}_{\mathrm{i}}^{\text {new }}, \mathrm{IMG}, \mathrm{R}_{\mathrm{i}}\right\}$

\section{Security Analysis of the Proposed Scheme}

In this section, we have analyzed the security features of our proposed scheme.

$\checkmark \quad$ Stolen smart card Attack: An adversary may get a lost or stolen smart card of a user and may extract the information, $\left\{\mathrm{B}_{\mathrm{i}}, \mathrm{C}_{\mathrm{i}}, \mathrm{D}_{\mathrm{i}}, \mathrm{IMG}, \mathrm{R}_{\mathrm{i}}\right\}$, stored in it. However, the adversary will not be able to extract the relevant information $A_{i}$ from $B_{i}$ as it is protected with password $\mathrm{PW}_{\mathrm{i}}$ and identity $\mathrm{ID}_{\mathrm{i}}$ of the user.

$\mathrm{B}_{\mathrm{i}}=\mathrm{A}_{\mathrm{i}} \oplus \mathrm{U}_{2}$, where $\mathrm{U}_{2}=\mathrm{h}\left(\mathrm{PW}_{\mathrm{i}} \oplus \mathrm{ID}_{\mathrm{i}}\right)$.

The shared secret PSK, used for generating authentication message, is also well protected. The adversary without PSK and $\mathrm{A}_{\mathrm{i}}$ will not be able to generate any login message. If the adversary attempts to generate the session key, they will not succeed as $A_{i}$ is required.

$\checkmark$ Impersonation Attack: To impersonate as a legal user, the adversary must be able to generate the login messages $\left\{\mathrm{M}_{1}, \mathrm{M}_{2}, \mathrm{M}_{\mathrm{v}}\right\}$. The adversary must possess the user information $\mathrm{A}_{\mathrm{i}}, \mathrm{ID}_{\mathrm{i}}$, PSK. A registered but malicious user can use its PSK for login message generation but $A_{i}$ is unknown to it. Also, in the smart card, $A_{i}$ is protected with the user password and the identity, $B_{i}=A_{i} \oplus U_{2}$, where $U_{2}=h\left(P_{i} \oplus I_{i}\right)$.

$\checkmark$ Server Masquerade Attack: The server identity $\operatorname{SID}_{\mathrm{j}}$ is publicly known, so the probability of server masquerading increases. An adversary may intercept all the messages intended for the server $\operatorname{SID}_{j}$. However, the adversary, a malicious server, will not be able to extract any information from the captured messages, as they will not have the server secret $\mathrm{h}\left(\mathrm{SID}_{\mathrm{j}} \| \mathrm{PSK}\right)$. Moreover, if the adversary is a registered user, they can generate $\mathrm{h}\left(\mathrm{SID}_{\mathrm{j}} \| \mathrm{PSK}\right)$ but they will not have any knowledge about $\mathrm{X}_{\mathrm{s}}$.

$\checkmark$ Offline password guessing attack: The adversary may use a stolen smart card to extract all the information, $\mathrm{SC}_{\mathrm{i}}=\left\{\mathrm{B}_{\mathrm{i}}, \mathrm{C}_{\mathrm{i}}, \mathrm{D}_{\mathrm{i}}, \mathrm{IMG}, \mathrm{R}_{\mathrm{i}}\right\}$, stored in it. Now the adversary try to guess the password by selecting the blocks of image IMG. But the adversary is unaware of the number of blocks to select from the image.

$\checkmark$ Man-in-the-middle attack: In this attack, an adversary eavesdrops a communication and tries to extract the information to generate the session key. The adversary intercepts the user's login messages, $\left\{\mathrm{M}_{1}, \mathrm{M}_{2}, \mathrm{M}_{\mathrm{v}}\right\}$, and attempts to extract the parameters for session key generation. However, this will not be successful as the adversary is unaware of the server secrets $\mathrm{h}\left(\mathrm{SID}_{\mathrm{j}} \| \mathrm{PSK}\right)$ and $\mathrm{X}_{\mathrm{s}}$, which are required to 
extract $I_{i}$ from $M_{1}$. Without the $I_{i}$, the adversary will not be able to compute $A_{i}, N_{i}$, used in session key.

$\checkmark \quad$ Replay Attack: The adversary may eavesdrop an ongoing communication and stores the login messages, $\left\{\mathrm{M}_{1}, \mathrm{M}_{2}, \mathrm{M}_{\mathrm{v}}\right\}$. They can, in future, use these message for performing replay attack. However, this will not be successful, as the adversary will not be able to generate a valid authentication message.

$\checkmark$ Insider Attack: The user during registration does not provide the password in its original form. The password is hashed with a random number $R_{i}$ for $U_{1}$ and for $U_{2}$, it is hashed with the user's identity $\mathrm{ID}_{\mathrm{i}}$ as: $\mathrm{U}_{1}=\mathrm{h}\left(\mathrm{R}_{\mathrm{i}} \| \mathrm{PW}_{\mathrm{i}}\right)$ and $\mathrm{U}_{2}=\mathrm{h}\left(\mathrm{PW}_{\mathrm{i}} \oplus \mathrm{ID}_{\mathrm{i}}\right)$. Hence, making the proposed scheme resistant against this attack.

\section{Performance Analysis}

In this section, we have compared the security performance of our scheme with other existing authentication schemes which is shown in the Table 2.

Table 2. Comparison of Security Attributes of Our Proposed Scheme with Existing Scheme

\begin{tabular}{|c|c|c|c|c|}
\hline $\begin{array}{c}\text { Security } \\
\text { Attributes }\end{array}$ & Proposed scheme & $\begin{array}{c}\text { Mishra } \text { et al. } \\
{[\mathbf{1 7}]}\end{array}$ & $\begin{array}{c}\text { Chuang and Chen } \\
{[\mathbf{1 6}]}\end{array}$ & $\begin{array}{c}\text { Li and Hwang } \\
{[\mathbf{1 0}]}\end{array}$ \\
\hline $\mathbf{A 1}$ & Yes & Yes & Yes & No \\
\hline $\mathbf{A 2}$ & Yes & Yes & Yes & Yes \\
\hline $\mathbf{A 3}$ & Yes & Yes & Yes & Yes \\
\hline A4 & Yes & Yes & Yes & No \\
\hline A5 & Yes & No & No & No \\
\hline A6 & Yes & No & No & Yes \\
\hline A7 & Yes & No & No & No \\
\hline A8 & Yes & Yes & No & No \\
\hline A9 & Yes & Yes & Yes & No \\
\hline A10 & Yes & Yes & Yes & No \\
\hline
\end{tabular}

A1: User anonymity; A2: Human Participation; A3: Simple Password Change; A4: Mutual Authentication; A5: Resistance against impersonation attack; A6: Resistance against server spoofing; A7: Resistance against Stolen Smart card attack; A8: Resistance against Man-in-the-middle attack; A9: Resistance against Insider Attack; A10: Session Key verification.

The computational cost of the authentication scheme depends on the hash function and the operations performed. The following notations are used for computing the computational complexity of the proposed scheme:

$\mathrm{T}_{\mathrm{h}}$ : time for executing a one-way hash function $\mathrm{h}($.$) .$

$\mathrm{T}_{\mathrm{x}}$ : time for executing exclusive - OR operation.

$\mathrm{T}_{\mathrm{c}}$ : time required for executing comparison operation.

Table 3. Performance Comparison with Existing Scheme

\begin{tabular}{|c|c|c|c|c|}
\hline Phase & $\begin{array}{c}\text { Proposed } \\
\text { Scheme }\end{array}$ & $\begin{array}{c}\text { Mishra } \text { et al. } \\
{[17]}\end{array}$ & $\begin{array}{c}\text { Chuang and Chen } \\
{[16]}\end{array}$ & $\begin{array}{c}\text { Li and Hwang } \\
{[10]}\end{array}$ \\
\hline P1 & $5 T_{h}+3 T_{x}$ & $7 T_{h}+5 T_{x}$ & $3 T_{h}+3 T_{x}$ & $3 T_{h}+T_{x}$ \\
\hline P2 & $6 T_{h}+5 T_{x}+T_{c}$ & $6 T_{h}+6 T_{x}+T_{c}$ & $4 T_{h}+3 T_{x}+T_{c}$ & $T_{h}+2 T_{x}+T_{c}$ \\
\hline P3 & $9 T_{h}+6 T_{x}+2 T_{c}$ & $12 T_{h}+5 T_{x}+3 T_{c}$ & $13 T_{h}+6 T_{x}+3 T_{c}$ & $5 T_{h}+4 T_{x}+2 T_{c}$ \\
\hline P4 & $8 T_{h}+6 T_{x}+T_{c}$ & $5 T_{h}+3 T_{x}+T_{c}$ & $2 T_{h}+5 T_{x}+T_{c}$ & $3 T_{h}+2 T_{x}+T_{c}$ \\
\hline Total & $28 T_{h}+20 T_{x}+4 T_{c}$ & $30 T_{h}+19 T_{x}+5 T_{c}$ & $22 T_{h}+17 T_{x}+5 T_{c}$ & $12 T_{h}+9 T_{x}+4 T_{c}$ \\
\hline
\end{tabular}


P1: Registration Phase; P2: Login Phase; P3: Authentication Phase; P4: Password Change Phase

Table 3 shows that the computational cost of the proposed scheme is relatively lower than Mishra et al.'s [17] scheme but with higher security. We can conclude that the security can be enhanced by affording a little bit of extra cost.

\section{Conclusion}

In this paper, we have proposed a user-friendly authentication scheme using smart card where the users need not to spend any extra effort to remember any alphanumeric complicated password for the assurance of higher security. Our proposed scheme not only uses the graphic-based password but also can provide the security to a quality level at lower cost. Therefore, our scheme is well suited for practical implementation for all kind of users even though they are not expertise in computer.

\section{References}

[1] L. Lamport, "Password authentication with insecure communication," Communications of the ACM, vol. 24, no. 11, pp. 770-772, 1981.

[2] N. M. Haller, "The S/KEY one-time password system," RFC1760, February 1995.

[3] G. Horng, "Password authentication without using a password table," Information Processing Letters, vol. 55, no. 5, pp. 247-250, 1995.

[4] M.-S. Hwang and L.-H. Li, "A new remote user authentication scheme using smart cards," IEEE Transactions on Consumer Electronics, vol. 46, no.1, pp. 28-30, 2000.

[5] C.-T. Li, C.-C. Lee, C.-J. Liu, and C.-W. Lee, "A robust remote user authentication scheme against smart card security breach," Proceedings of Data and Applications Security and Privacy XXV, vol. 6818, pp. 231-238, 2011.

[6] S. S. Sonwanshi, R. R. Ahirwal, and Y. K. Jain, "An efficient smart card based remote user authentication scheme using hash function," in Proceedings of the IEEE Students' Conference on Electrical, Electronics and Computer Science (SCEECS '12), pp.1-4, March 2012.

[7] R. Madhusudhan, R. C. Mittal, "Dynamic Id-based remote user password authentication schemes using smart cards: A review", Journal of Network and Computer Application, vol. 35, no. 4, pp. 1235-1248, 2012.

[8] J.-Y. Liu, A.-M. Zhou, and M.-X. Gao, "A new mutual authentication scheme based on nonce and smart cards," Computer Communications, vol. 31, no. 10, pp. 2205-2209, 2008.

[9] M. K. Khan, "Fingerprint biometric-based self-authentication and deniable authentication schemes for the electronic world," IETE Technical Review, vol. 26, no. 3, pp. 191-195, 2009.

[10] C.-T. Li and M.-S. Hwang, "An efficient biometrics-based remote user authentication scheme using smart cards," Journal of Network and Computer Applications, vol.33, no.1, pp.1-5, 2010.

[11] X. Li, J.W. Niu, J. Ma, W.D. Wang, and C.L. Liu, "Cryptanalysis and improvement of a biometric-based remote authentication scheme using smart cards". Journal of Network and Computer Applications, vol. 34, pp. 73-79, 2011.

[12] A.K Das, "Analysis and improvement on an efficient biometric based remote user authentication scheme using smart cards". IET Information Security, vol. 5, no. 3, pp. 145-151, 2011.

[13] V.C Sekhar, and Mrudula.S. "Secure and Efficient Biometric Based Remote User Authentication Scheme Using Smart Cards" ICECIT, Dec 21, SRIT, India, 2012.

[14] Y. An, "Security analysis and enhancements of an effective biometric-based remote user authentication scheme using smart cards," Journal of Biomedicine and Biotechnology, vol. 2012, 2012.

[15] M. K. Khan, and S. Kumari, "An Improved Biometrics-Based Remote User Authentication Scheme with User Anonymity", BioMed Research International, Hindawi Publishing Corporation, 2013.

[16] M.-C. Chuang and M. C. Chen, "An anonymous multi-server authenticated key agreement scheme based on trust computing using smart cards and biometrics." Experts Systems with Applications, Vol. 41, No. 4, pp. 1411-1418, 2014.

[17] D. Mishra, A. K. Das and S. Mukhopadhyay, "A secure user anonymity-preserving biometric-based multi-server authenticated key agreement scheme using smart cards.", Expert Systems with Applications, vol. 41, no. 18, pp. 8129-8143, 2014.

[18] T. Maitra, D. Giri, “An Efficient Biometric and Password-Based Remote User Authentication using Smart Card for Telecare Medical Information Systems in Multi-Server Environment." Journal of Medical Systems, vol. 38, no. 12, 2014 


\section{Authors}
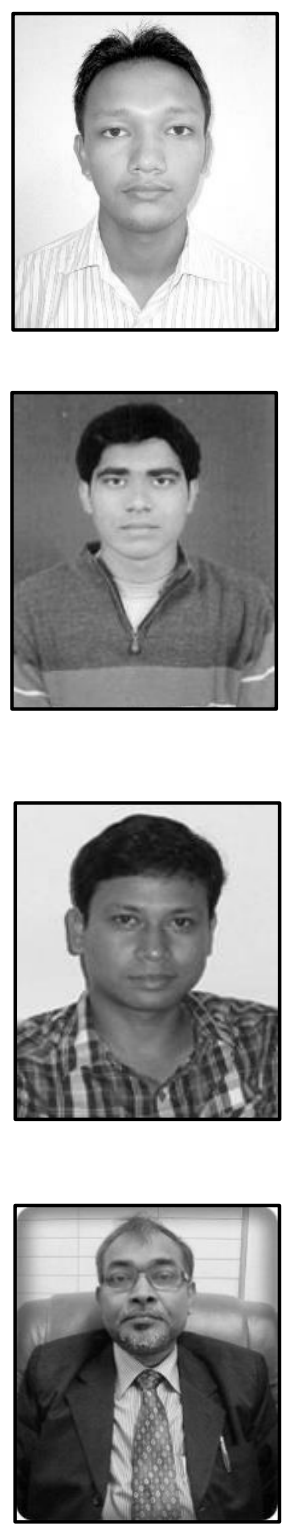

Chandan Tilak Bhunia, he did his B. Tech. in Radiophysics and Electronics in 1983 from Calcutta University. He received his M. Tech. in Radiophysics and Electronics in 1985 and then joined North Bengal University as a lecturer of Computer Science \& Applications in 1988. He became Assistant Professor of ECE at NERIST, Govt. of India in 1990. He got P. hd. in Computer Science \& Engineering from Jadavpur University. He became a full Professor in 1997 at NERIST. Currently, he is working as a Director of National Institute of Technology, Arunachal Pradesh. He has published around 150 research papers in various national and international journals of repute. Under his supervision, five Ph.D. scholars got awarded and nine scholars are currently working in various fields. 